\title{
Mitochondrial protein import: differential recognition of various transport intermediates by antibodies
}

\author{
Thomas Söllner, Nikolaus Pfanner and Walter Neupert \\ Institut für Physiologische Chemie, Universität München, Goethestrasse 33, D-8000 München 2, FRG
}

Received 4 January 1988

\begin{abstract}
The precursors of the mitochondrial proteins ADP/ATP carrier (AAC) and $F_{1}-A T P a s e$ subunit $\beta\left(F_{1} \beta\right)$ were accumulated at the stages of binding to receptor sites on the mitochondrial outer membrane, or in contact sites between outer and inner membranes. Specific antibodies raised against the mature proteins were added to the isolated mitochondria and efficiently bound to these translocation intermediates. Further movement of the precursors to consecutive steps along their import pathway was thereby inhibited. Controls showed that precursor proteins which were inserted into or translocated across the outer membrane were not recognized by the antibodies unless the mitochondrial membranes were disrupted. We conclude that the trapped translocation intermediates have antigenic sites exposed to the outside of the outer membrane.
\end{abstract}

Mitochondria; Protein import; ADP/ATP carrier; $\mathrm{F}_{1}$-ATPase subunit $\beta$

\section{INTRODUCTION}

Analysis of translocation intermediates of mitochondrial precursor proteins has provided important information on the mechanisms of mitochondrial protein import [1-18]. For example, the trapping of the $F_{1} \beta$ precursor protein in contact sites between outer and inner mitochondrial membranes (see fig.3A) demonstrated the essential role of these sites for protein import $[7,13,15,17,18]$. The binding of AAC to the mitochondrial surface was resolved into two distinct steps including interaction with initial binding sites (stage 2 intermediate) followed by insertion into the outer membrane (stage 3 intermediate) (see fig.3B) [12,13].

Translocation intermediates have been generated by lowering the temperature or by decreasing the levels of ATP in the import reactions. This was performed in either the presence or absence of a mitochondrial membrane potential. Accessibility

Correspondence address: T. Söllner, Institut für Physiologische Chemie, Universität München, Goethestrasse 33, D-8000 München 2, FRG for proteases added to the isolated mitochondria served as a criterion that the contact-site intermediate of $F_{1} \beta$ and the stage 2 intermediate of AAC had parts exposed to the outside of the outer membrane $[7,12,13]$. Furthermore, the $F_{1} \beta$ intermediate could be generated by employing a precursor protein which had antibodies pre-bound to the mature protein part $[7,17]$. With the latter method, however, the amount of translocation intermediates generated was markedly decreased when compared to the two other methods (low temperature or low levels of ATP). We show here that the contact-site intermediate of $\mathrm{F}_{1} \beta$ and the stage 2 intermediate of AAC, which are generated at low levels of ATP, are efficiently recognized by antibodies added after the transfer in vitro. The precursor proteins can thereby be arrested in the intermediate location.

\section{MATERIALS AND METHODS}

Isolation of Neurospora crassa mitochondria, synthesis of precursor proteins in rabbit reticulocyte lysates [19] in the presence of $\left[{ }^{35}\right.$ S]methionine, in vitro import reactions, and generation of translocation intermediates were performed as described $[12,13]$. Mitochondria were re-isolated after the im- 
port reactions and resuspended in buffer containing $250 \mathrm{mM}$ sucrose, $80 \mathrm{mM} \mathrm{KCl}, 5 \mathrm{mM} \mathrm{MgCl}_{2}, 3 \%$ (w/v) bovine serum albumin, and $10 \mathrm{mM}$ Mops, adjusted to $\mathrm{pH} 7.2$ with $\mathrm{KOH}$, in the presence of apyrase $(0.25 \mathrm{U} / \mathrm{ml})$, antimycin $A(8 \mu \mathrm{M})$, and oligomycin $(20 \mu \mathrm{M})$. Antibodies against purified $\mathrm{F}_{1} \beta$ and $\mathrm{AAC}$ were added. After $30 \mathrm{~min}$ at $0^{\circ} \mathrm{C}$, mitochondria were re-isolated and lysed in buffer containing $1 \%$ Triton $X-100$. The antibodyprotein complexes were harvested using protein A-Sepharose as in [7]. Analysis of the immunoprecipitates by SDSpolyacrylamide gel electrophoresis [20], and fluorography of the gels [21] were performed as published [6]. Results were quantified by densitometry of the X-ray films.

\section{RESULTS}

At low levels of ATP, mitochondrial precursor proteins are not sufficiently unfolded to be competent for complete import into mitochondria [13]. They are only partially inserted into the mitochondrial membranes. Under these conditions, $F_{1} \beta$, for instance, can be accumulated in translocation contact sites in the presence of a mitochondrial membrane potential $[13,15]$. The precursor of $F_{1} \beta$ becomes proteolytically processed at its aminoterminus by the processing peptidase in the mitochondrial matrix, generating mature-sized $\mathrm{F}_{1} \beta$ $\left(m-F_{1} \beta\right)$. A major portion of the molecule, however, is still outside the outer membrane since it is accessible to externally added proteases $[7,13,17]$. This species of $m-F_{1} \beta$ thus spans both mitochondrial membranes.

In order to generate low levels of ATP, first the endogenous ATP present in the in vitro import system (isolated Neurospora mitochondria and rabbit reticulocyte lysate containing radiolabelled mitochondrial precursor proteins) was degraded by pre-incubation with apyrase, an ATPase and ADPase from potato [22]. Low levels of ATP were then re-established by the addition of ADP which led to formation of ATP via adenylate kinase $[13,23]$.

In the experiment described in fig. $1 \mathrm{~A}, \mathrm{~B}, \mathrm{~F}_{1} \beta$ was accumulated in translocation contact sites. The mitochondria were then re-isolated, lysed in buffer containing $1 \%(\mathrm{w} / \mathrm{v})$ Triton $\mathrm{X}-100$, and $\mathrm{F}_{1} \beta$ was immunoprecipitated using antibodies directed against mature $F_{1} \beta$ and harvested with protein $A-$ Sepharose. The immunoprecipitates were analyzed by SDS-polyacrylamide gel electrophoresis and fluorography of the dried gels. Mature-sized $F_{1} \beta$ $\left(\mathrm{m}-\mathrm{F}_{1} \beta\right)$ was quantified by densitometry of the $\mathrm{X}$ ray films. Of the total $m-F_{1} \beta$ which was associated
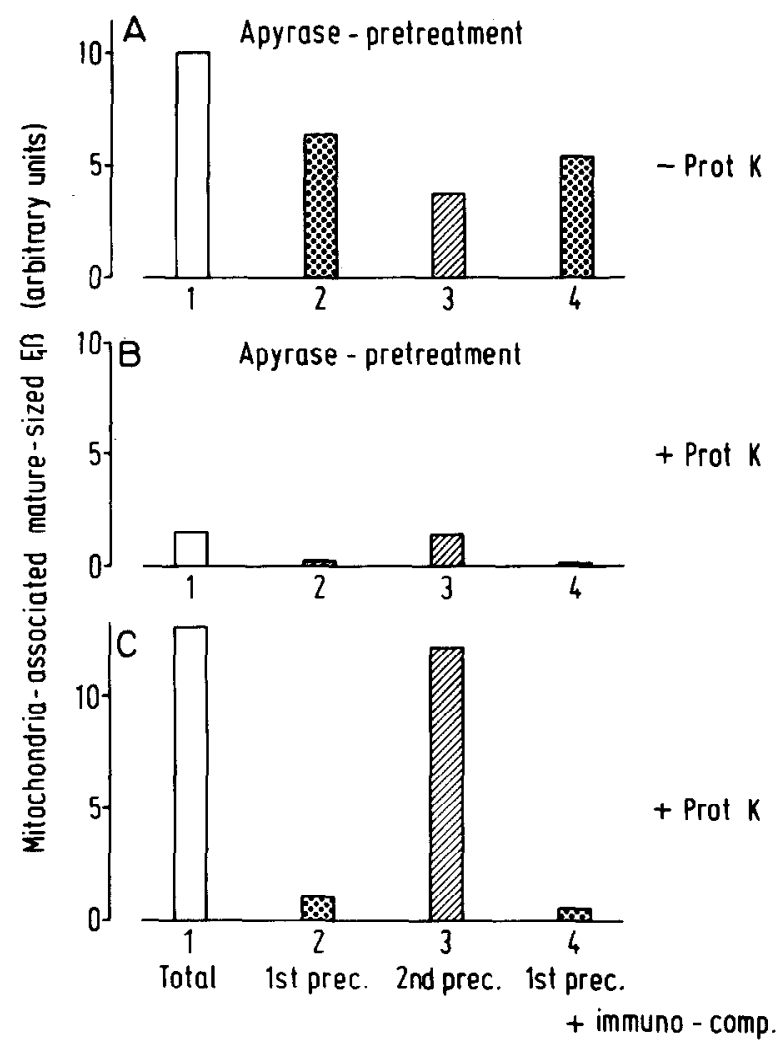

Fig.1. $\mathbf{F}_{1} \beta$ accumulated in translocation contact sites is accessible to antibodies added to isolated mitochondria. In experiment A, isolated Neurospora mitochondria and rabbit reticulocyte lysates containing radiolabelled mitochondrial precursor proteins were pre-treated with apyrase $(0.7 \mathrm{U} / \mathrm{ml})$ as described [22]. The mitochondria were added to the reticulocyte lysate in the presence of $8 \mathrm{mM}$ ascorbate, $0.2 \mathrm{mM}$ $N, N, N^{\prime}, N^{\prime}$-tetramethylphenylenediamine (TMPD), and $6 \mathrm{mM}$ ADP as in $[12,13]$. After $20 \mathrm{~min}$ at $25^{\circ} \mathrm{C}$, mitochondria were reisolated. Mitochondria from one aliquot were lysed in $1 \%(\mathrm{w} / \mathrm{v})$ Triton $X-100$, and $F_{1} \beta$ was immunoprecipitated (sample 1). With a second aliquot, the re-isolated mitochondria were incubated with antibodies against $\mathrm{F}_{1} \beta\left(30 \mathrm{~min}\right.$ at $\left.0^{\circ} \mathrm{C}\right)$. Mitochondria were re-isolated, lysed in $1 \%$ Triton X-100 and antibody $-\mathrm{F}_{1} \beta$ complexes were harvested, after addition of protein A-Sepharose, by centrifugation. Radiolabelled $\mathrm{m}-\mathrm{F} \beta$ was determined as described in section 2 (sample 2). The supernatant of this reaction ('1st prec.') again received antibodies against $F_{1} \beta$ and a second immunoprecipitation was performed ('2nd prec.') (sample 3). Reaction 4 was performed as described for sample 2 except that the mitochondria were lysed in the presence of an excess of unlabelled mitochondria ('immunocomp.'). Experiment B was performed as described for A except that the mitochondria were treated with proteinase $\mathrm{K}(20 \mu \mathrm{g} / \mathrm{ml}$; Prot $\mathrm{K})$ after the import incubation as described [12]. Experiment $C$ was performed as described for B except that the pre-treatment with apyrase and the addition of ADP were omitted, and that the import incubation was performed for $40 \mathrm{~min}$ at $25^{\circ} \mathrm{C}$. 
with mitochondria (fig.1A, column 1), 15\% was protected against externally added proteinase $K$ (fig. $1 \mathrm{~B}$, column 1). Thus, $85 \%$ of $\mathrm{m}-\mathrm{F}_{1} \beta$ in column 1 of fig.1A was trapped in translocation contact sites, i.e. was accessible to the matrix-located processing peptidase and to externally added proteases $[7,13,15,17]$. In a parallel reaction, the mitochondria with $m-F_{1} \beta$ trapped in the two-membranespanning fashion were re-isolated and incubated with antibodies against $\mathrm{F}_{1} \beta$ for $30 \mathrm{~min}$ at $0^{\circ} \mathrm{C}$. The mitochondria were re-isolated and lysed in the presence of $1 \%(\mathrm{w} / \mathrm{v})$ Triton $\mathrm{X}-100$. The antibodyprotein complexes were then harvested using protein A-Sepharose (fig. 1A, column 2). The supernatant of the immunoprecipitation was incubated with $F_{1} \beta$-antibodies bound to protein A-Sepharose (fig. $1 A$, column 3 ). By doing so, $F_{1} \beta$ which had not been recognized by the antibodies in the incubation with intact mitochondria was immunoprecipitated. About $65 \%$ of $m-F_{1} \beta$ was recognized by the antibodies in the first incubation (fig.1A, column 2), the remaining $35 \%$ being recognized in the second incubation with antibodies. As a control, lysis of mitochondria with Triton X-100 was performed in the presence of an excess amount of unlabelled $F_{1} \beta$ such that the binding capacity of the antibodies was exceeded more than 10-fold. Nearly the same amount of $\mathrm{m}$ $F_{1} \beta$ was recognized (fig.1A, column 4) compared to the samples not having unlabelled $F_{1} \beta$ present (fig.1A, column 2). It can therefore be excluded that the $\mathrm{m}-\mathrm{F}_{1} \beta$ seen in column 2 of fig. $1 \mathrm{~A}$ was recognized after the lysis of mitochondria by antibodies which might have been unspecifically adsorbed to mitochondria.

In the experiment described in fig. $1 \mathrm{C}, \mathrm{F}_{1} \beta$ was imported into the mitochondrial matrix in the presence of high levels of ATP. $F_{1} \beta$ that was not completely translocated was removed by addition of proteinase $\mathrm{K}$ after import. Practically all of the completely imported $F_{1} \beta$ was recognized only when antibodies were added to lysed mitochondria (fig.1C, columns 1,3). This excluded that antibodies added to intact mitochondria could penetrate into the mitochondria (fig.1C, columns $2,4)$. As expected, the protease-protected $m-F_{1} \beta$ obtained in the experiment of fig.1B was only recognized when antibodies were added to lysed mitochondria (fig.1B, columns 1,3 ). This imported $\mathrm{F}_{1} \beta$ is included in the $35 \%$ of $\mathrm{m}-\mathrm{F}_{1} \beta$ in fig. $1 \mathrm{~A}$ which are not recognized by antibodies added to intact mitochondria.

In summary, we conclude that $m-F_{1} \beta$ accumulated in translocation contact sites can be efficiently (by more than $75 \%$ ) recognized by specific antibodies added to intact mitochondria. Imported $F_{1} \beta$ is not recognized by the antibodies under these conditions. It should be emphasized that the arresting of translocation intermediates shown here is different from the trapping of precursor proteins with pre-bound antibodies [7]. In the latter approach, the import efficiency of the modified (i.e. antibody-bound) precursors is decreased. Antibody binding after accumulation

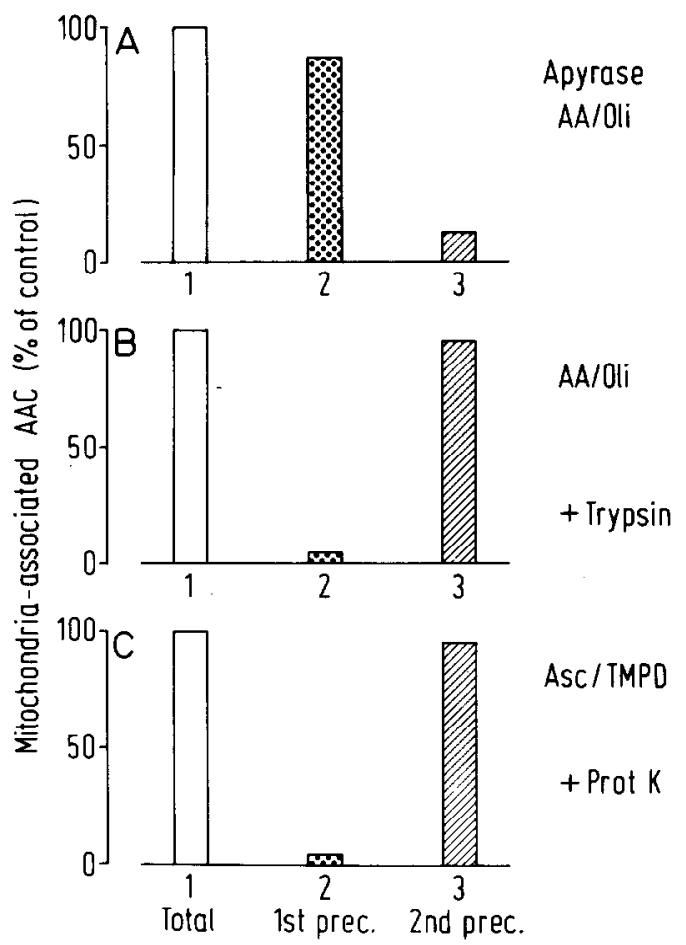

Fig.2. The stage 2 intermediate of $\mathrm{AAC}$ is recognized by antibodies added to isolated mitochondria. Experiment $\mathbf{A}$ was performed as described in the legend to fig.1A, samples 1-3, with the following modifications: antimycin $A(8 \mu \mathrm{M})$ and oligomycin $(20 \mu \mathrm{M})(\mathrm{AA} / \mathrm{Oli})$ were added. Ascorbate, TMPD, and ADP were omitted. Incubation was for $25 \mathrm{~min}$ at $25^{\circ} \mathrm{C}$. Antiserum raised against AAC was employed. Experiment B was performed as described for $A$ except that pre-treatment with apyrase was omitted and that the mitochondria were treated with trypsin $(20 \mu \mathrm{g} / \mathrm{ml})$ after the binding reaction as described [12]. Experiment $C$ was performed as described for $B$ except that $8 \mathrm{mM}$ ascorbate (Asc) and $0.2 \mathrm{mM}$ TMPD were added instead of antimycin $A$ and oligomycin, and that proteinase $\mathrm{K}(200 \mu \mathrm{g} / \mathrm{ml}$; Prot $\mathrm{K})$ was used instead of trypsin. 
of precursors in translocation contact sites has two important advantages: the unmodified authentic precursor can be used, and the efficiency of trapping is high.

For the experiment described in fig.2, the precursor of AAC was accumulated at distinct steps of its import pathway into mitochondria as described previously $[12,13]$. In the absence of ATP and in the absence of a membrane potential, AAC binds to the mitochondrial surface where it remains accessible to externally added trypsin (stage 2 intermediate; fig.2A). In the absence of a membrane potential, but in the presence of ATP, $\mathrm{AAC}$ inserts into the outer membrane where it is protected against trypsin (stage 3 intermediate, fig.2B). In the presence of both ATP and a membrane potential, AAC is completely imported into the inner membrane and assembles to the mature form which is protected against even high concentrations of proteinase $\mathrm{K}$ (fig. $2 \mathrm{C}$ ).

Columns 1 of fig. 2 represent the total amount of AAC accumulated at the distinct import stages. In parallel reactions, the mitochondria having AAC associated with them were re-isolated and incubated with antibodies against mature AAC (which had been denatured by heating to $95^{\circ} \mathrm{C}$ in the presence of SDS prior to immunization). Of the stage 2 intermediates, $85-90 \%$ were recognized by the antibodies added to intact mitochondria (fig.2A, column 2). The remaining $10 \%$ were only recognized when antibodies were added to detergent-lysed mitochondria (fig.2A, column 3). The stage 3 intermediate (fig.2B, column 3) and the assembled AAC (fig.2C, column 3) were only accessible to antibodies in lysed mitochondria.

Controls showed that at least $90 \%$ of the AAC precursors bound to apyrase-treated mitochondria (fig.2A) were true stage 2 intermediates, i.e. were on the correct assembly pathway (Pfaller, Pfanner and Neupert, in preparation). AAC accumulated at stage 2 can be transported to stage 3 after addition of nucleoside triphosphates, and then be translocated into the inner membrane after reestablishing a membrane potential [13]. When antibodies were bound to the stage 2 intermediates, however, further transport of AAC into the mitochondrial membranes did not occur (not shown). Thus, the binding of antibodies, as described here, led to stable arrest of the stage 2 intermediate of AAC.
In summary, the bulk of the stage 2 intermediates of AAC can be recognized by antibodies added to isolated mitochondria. AAC which is inserted into the outer membrane (stage 3 intermediate) or inner membrane (assembled AAC) is not accessible to externally added antibodies unless the mitochondria are lysed.

\section{DISCUSSION}

We report here that translocation intermediates of mitochondrial precursor proteins, which are
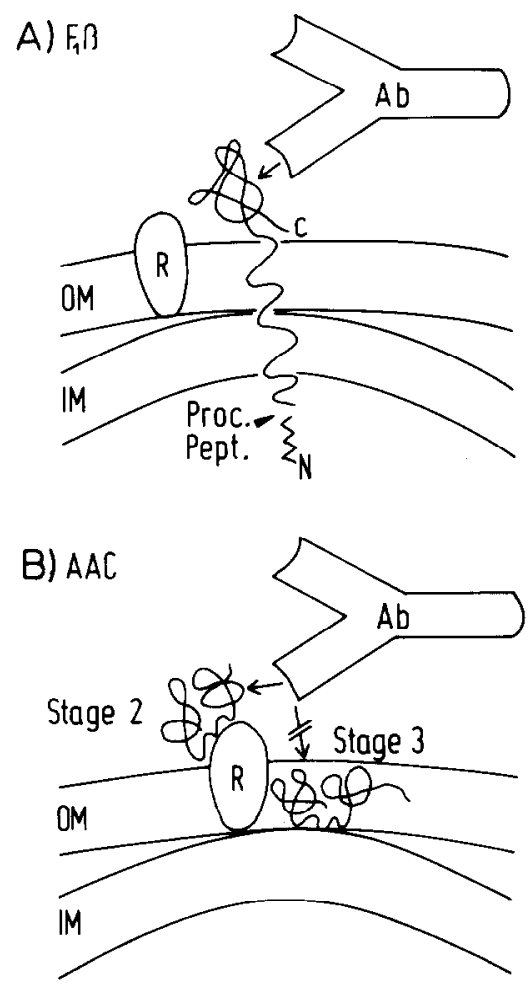

Fig.3. Working model on the accessibility of externally added antibodies to translocation intermediates of mitochondrial precursor proteins (modified after $[7,12,13,24]$ ). (A) $F_{1} \beta$ is accumulated in contact sites between outer (OM) and inner (IM) mitochondrial membranes. The amino-terminal (N) presequence is cleaved off by the processing peptidase (Proc. Pept.) located in the mitochondrial matrix. Other, probably carboxyl-terminal (C), portions of $F_{1} \beta$ are outside the outer membrane and can be recognized by externally added antibodies (Ab). $R$, receptor. (B) The stage 2 intermediate of AAC interacts with receptor sites (R) on the mitochondrial surface and is accessible to externally-added antibodies (Ab). The stage 3 intermediate is beyond the initial binding site. It is inserted into the outer membrane (OM) where it is not accessible to antibodies. 
trapped at the level of binding to receptor sites or in contact sites between both mitochondrial membranes, are accessible to externally added antibodies (summarized in fig.3). These findings are relevant with regard to approaches for accumulating translocation intermediates of mitochondrial precursor proteins in order to study details of import pathways. For example, binding of precursors to receptor sites and import via contact sites were mainly characterized by analyzing translocation intermediates (see section 1). It is therefore of considerable importance to define unambiguously the topological arrangement of such import intermediates. So far, accessibility of various proteases to the translocation intermediates has been employed as the major tool to study their topology. The binding of specific antibodies to intermediates now provides an efficient non-destructive method for this purpose. We conclude that the stage 2 intermediate of $\mathrm{AAC}$ and the contact-site intermediate of $F_{1} \beta$ have antigenic sites exposed to the outside of the outer membrane (fig.3).

Arresting import intermediates by binding of antibodies provides the possibility to visualize efficiently the intermediates by immunocytochemical techniques. The antibody-arrested translocation intermediates may be used as marker proteins for purifying receptors and contact sites. Furthermore, preparation of specific antibodies against distinct portions of the precursor proteins and investigating the ability of these antibodies to bind to the trapped intermediates will reveal which portions of the intermediate proteins are exposed to the outside of the outer membrane.

Acknowledgements: We thank Christine Forster for excellent technical assistance. We are grateful to Drs Franz-Ulrich Hartl and Donald W. Nicholson for critically reading the manuscript. This work was supported by the Sonderforschungsbereich 184 (project B1) and the Fonds der Chemischen Industrie.

\section{REFERENCES}

[1] Hennig, B. and Neupert, W. (1981) Eur. J. Biochem. 121, 203-212.

[2] Hennig, B., Köhler, H. and Neupert, W. (1983) Proc. Natl. Acad. Sci. USA 80, 4963-4967.

[3] Zwizinski, C. and Neupert, W. (1983) J. Biol. Chem. 258, $13340-13346$.

[4] Zwizinski, C., Schleyer, M. and Neupert, W. (1983) J. Biol. Chem. 258, 4071-4074.

[5] Riezman, H., Hay, R., Witte, C., Nelson, N. and Schatz, G. (1983) EMBO J. 2, 1113-1118.

[6] Pfanner, N. and Neupert, W. (1985) EMBO J. 4, 2819-2825.

[7] Schleyer, M. and Neupert, W. (1985) Cell 43, 339-350.

[8] Schmidt, B., Pfaller, R., Pfanner, N., Schleyer, M. and Neupert, W. (1985) in: Achievements and Perspectives of Mitochondrial Research (Quagliariello, E. et al. eds) vol.II, pp.389-396, Elsevier, Amsterdam, New York.

[9] Hartl, F.-U., Schmidt, B., Wachter, E., Weiss, H. and Neupert, W. (1986) Cell 47, 939-951.

[10] Hartl, F.-U., Ostermann, J., Guiard, B. and Neupert, W. (1987) Cell, in press.

[11] Pfaller, R. and Neupert, W. (1987) EMBO J. 6, 2635-2642.

[12] Pfanner, N. and Neupert, W. (1987) J. Biol. Chem. 262, 7528-7536.

[13] Pfanner, N., Tropschug, M. and Neupert, W. (1987) Cell 49, 815-823.

[14] Pfanner, N., Müller, H.K., Harmey, M.A. and Neupert, W. (1987) EMBO J. 6, 3449-3454.

[15] Pfanner, N., Hartl, F.-U., Guiard, B. and Neupert, W. (1987) Eur. J. Biochem. 169, 289-293.

[16] Pfanner, N., Hoeben, P., Tropschug, M. and Neupert, W. (1987) J. Biol. Chem. 262, 14851-14854.

[17] Schwaiger, M., Herzog, V. and Neupert, W. (1987) J. Cell Biol. 105, 235-246.

[18] Chen, W.-J. and Douglas, M.G. (1987) Cell 49, 651-658.

[19] Pelham, H.R.B. and Jackson, R.J. (1976) Eur. J. Biochem. 67, 247-256.

[20] Laemmli, U.K. (1970) Nature 227, 680-685.

[21] Chamberlain, J.P. (1979) Anal. Biochem. 98, 132-135.

[22] Pfanner, N. and Neupert, W. (1986) FEBS Lett. 209, $152-156$.

[23] Frank, R., Trosin, M., Tomasselli, A.G., Schulz, G.E. and Schirmer, R.H. (1984) Eur. J. Biochem. 141, 629-636.

[24] Pfanner, N. and Neupert, W. (1987) Curr. Top. Bioenerg. 15, 177-219. 